\title{
Contribution of the space charge to the grain boundary energy in yttria-stabilized zirconia
}

\author{
Jun Wang • Hans Conrad
}

Received: 4 December 2013/ Accepted: 16 May 2014/Published online: 3 June 2014

(C) Springer Science+Business Media New York 2014

\begin{abstract}
A model based on the effect of a modest applied dc electric field on grain growth is proposed for the contribution of the space charge to the grain boundary (GB) energy in 3 mol \% yttria-stabilized zirconia (3 YSZ). The model considers that the total GB energy $\gamma_{b}^{0}$ (the capillary driving force for grain growth) consists of three major components: (a) $\gamma_{\mathrm{b}}^{\Sigma}$ due to the misorientation between neighboring grains, (b) $\gamma_{b}^{\mathrm{s}}$ due to the size misfit between the segregated solute and the solvent cations, and (c) $\gamma_{\mathrm{b}}^{\mathrm{e}}$ the electrostatic (space charge) component, which results from the segregation of the aliovalent yttrium ions to the grain boundaries. The former two components combined comprise $40 \%$ of the total GB energy in 3 YSZ and the electrostatic component $60 \%$. Based on the model, the calculated magnitudes of the three components were in qualitative accord with theoretical considerations and with values reported in the literature. A reduction in $\gamma_{b}^{e}$, and in turn in $\gamma_{\mathrm{b}}^{0}$, results from the bias exerted by the applied field on the space charge potential that occurs with the segregation of the yttrium ions to the grain boundaries. The observed reduction of grain growth in 3 YSZ by an applied electric field is attributed mainly to the reduction in $\gamma_{b}^{e}$ by the field.
\end{abstract}

\section{Introduction}

It is well-known that grain size (GS) is an important microstructure parameter in the processing and properties of ceramics [1]. It was discovered by Yang and Conrad [2]

J. Wang $\cdot$ H. Conrad $(\bowtie)$

Materials Science and Engineering Department, North Carolina

State University, Raleigh, NC 27695-7907, USA

e-mail: hans_conrad@ncsu.edu and subsequently confirmed by Ghosh et al. [3] that a modest applied dc electric field retards grain growth in 3 mol \% yttria-stabilized zirconia (3 YSZ). Subsequent work by Conrad and Yang [4, 5] established that the retarding effect increased with field strength up to $\sim 26 \mathrm{~V} /$ $\mathrm{cm}$ and then remained essentially constant to $\sim 60 \mathrm{~V} / \mathrm{cm}$, the effect being similar in the processing methods of sintering, annealing, and plastic deformation; see Fig. 1. Furthermore, the retarding effect by the field during sintering was found to be relatively independent of the relative density and in turn of the temperature and porosity. Based on these characteristics, it was proposed [4, 5] that the retardation of grain growth in 3 YSZ by an electric field results mainly from a reduction in the capillary driving force (namely, the grain boundary (GB) energy) by the interaction of the field with the space charge at the grain boundaries. Stereology studies gave that the field had no detectable influence on the grain shape, nor on the GS distribution [6, 7]. In this article, we develop a model for the retardation of grain growth in 3 YSZ by an electric field and perform isothermal annealing tests on $3 \mathrm{~mol} \%$ yttriastabilized tetragonal zirconia polycrystals (3Y-TZP) at 1300 and $1400{ }^{\circ} \mathrm{C}$ to ascertain its validity.

\section{The model}

It was now well-established that yttria segregates to the grain boundaries in polycrystalline yttria-stabilized zirconia (YSZ) [8-16]. A major factor proposed to be responsible for the segregation is the GB space charge. In view of the segregation, it is generally considered that the mechanism governing the grain growth rate in YSZ is "solute drag" [16]. Theoretical considerations give for this mechanism $[17,18]$ 


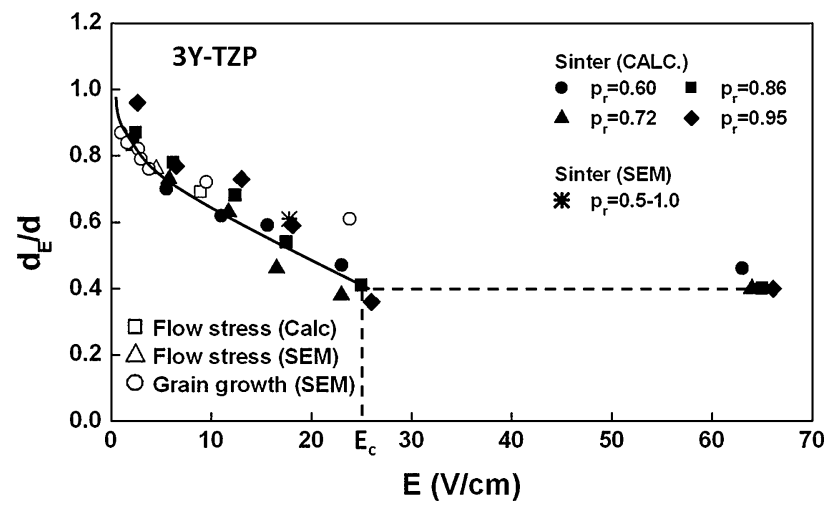

Fig. 1 The effect of applied dc electric field strength on the ratio of the grain size with field $\left(d_{\mathrm{E}}\right)$ to that without $(d)$ for various processing conditions. From Conrad and Di Yang [4]

$\dot{d}=P^{m} M=\frac{A}{k_{\mathrm{B}} T}\left(\frac{\gamma_{\mathrm{b}}^{0}}{r}\right)^{m} D$

where $\dot{d}$ is the grain growth rate, and $P=\gamma_{\mathrm{b}}^{0} / r$ is the capillary driving force, with $\gamma_{\mathrm{b}}^{0}$ the GB energy and $r=\alpha d$ $(\alpha \approx 0.5)$ the average GB radius of curvature. $M$ is the GB mobility, $A$ is a constant, $D$ is the diffusion coefficient of the rate-controlling ion, $k_{\mathrm{B}}$ is the Boltzmann's constant, and $m$ is the driving force exponent, which is given by the slope of a plot of $\log \dot{d}$ vs. $\log P$ at a constant temperature. The parameter " $m$ " depends on solute content and thus may vary with $P$ or $\dot{d}$. Employing Eq. 1, and assuming that a major effect of an applied electric field $E$ on $\dot{d}$ is through a reduction in $\gamma_{\mathrm{b}}^{0}[4,5]$, and dividing the grain growth rate $\dot{d}_{E}$ with an applied field by that without $(\dot{d})$, one obtains

$\frac{\dot{d}_{E}=\frac{A_{E} D_{E}}{k_{\mathrm{B}} T}\left[\frac{\left.\gamma_{\mathrm{b}}^{0}-\Delta \gamma_{\mathrm{b}}^{E}\right)}{d_{E}}\right]^{m_{E}}}{\dot{d}=\frac{A D}{k_{\mathrm{B}} T}\left[\frac{\gamma_{\mathrm{b}}^{0}}{d}\right]^{m}}$

where $\Delta \gamma_{\mathrm{b}}^{E}$ is the reduction in $\gamma_{\mathrm{b}}^{0}$ by the applied field $E$. The subscript $E$ in Eq. 2 refers to the parameter with applied field, its absence without field. In prior work [4, 5, 19], it was found that the parameters $A, D$, and $m$ are relatively independent of $E$. Rearranging Eq. 2 then gives for a constant temperature

$\Delta \gamma_{\mathrm{b}}^{E} \cong\left[1-\left(\dot{d}_{E} / \dot{d}\right)^{1 / m}\left(d_{E} / d\right)\right] \gamma_{\mathrm{b}}^{0}$

The magnitude of $\Delta \gamma_{\mathrm{b}}^{E}$ as a function of $E$ at a constant $T$ can then be obtained experimentally from a knowledge of $\gamma_{\mathrm{b}}^{0}$ and the effect of $E$ on the GS ratio $d_{E} / d$ at a constant grain growth rate $\dot{d}$, which then avoids the need to know the magnitude of the parameter $m$.

In our model, we take that $\gamma_{\mathrm{b}}^{0}$ in YSZ consists of the sum of three major components (see Fig. 2), namely

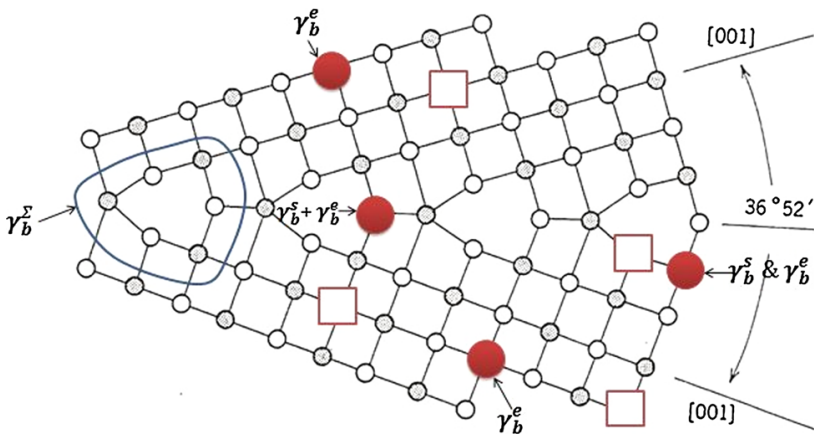

Fig. 2 Schematic of a large angle grain boundary in an ionic ceramic $(\mathrm{MgO})$ illustrating the three major contributions to the grain boundary energy. Dark shaded atoms are aliovalent solutes

$\gamma_{\mathrm{b}}^{0}=\gamma_{\mathrm{b}}^{\Sigma}+\gamma_{\mathrm{b}}^{\mathrm{s}}+\gamma_{\mathrm{b}}^{\mathrm{e}}$

where $\gamma_{b}^{\Sigma}$ results from the crystallographic misorientation between contacting grains, $\gamma_{b}^{s}$ from the ionic size misfit between the solute (yttrium) and solvent (zirconium) ions and $\gamma_{\mathrm{b}}^{\mathrm{e}}$ is the electrostatic (space charge) component, which results from the segregated, aliovalent yttrium ions at the GB.

A rough, theoretical estimate of $\gamma_{b}^{\Sigma}$ is [20]

$\gamma_{\mathrm{b}}^{\Sigma}=\lambda_{\mathrm{b}}^{\mathrm{c}} k_{\mathrm{B}} T / \Omega \quad\left(\mathrm{J} / \mathrm{m}^{2}\right)$

where $\lambda_{\mathrm{b}}^{\mathrm{c}}$ is the width of the GB core, $\Omega$ is the atomic volume of the host lattice, and $k_{\mathrm{B}}$ is Boltzmann's constant.

Regarding the solute ion size misfit component, estimates of the elastic strain energy $U[9,21]$ based on considerations by Eshelby [22, 23] are shown in Table 1; included are the calculated magnitudes of $U$ for 3Y-TZP. Taking into account, the number of solute ions in the GB core width one obtains for the size misfit energy per unit GB area

$\gamma_{\mathrm{b}}^{\mathrm{s}} \approx U \lambda_{\mathrm{b}}^{\mathrm{c}} C_{\mathrm{b}}^{*} / \Omega \quad\left(\mathrm{J} / \mathrm{m}^{2}\right)$

where $C_{\mathrm{b}}^{*}$ is the solute ion concentration segregated at the core.

Table 1 Estimates of the ionic size misfit energy $U$ in yttriastabilized zirconia

\begin{tabular}{llll}
\hline Authors & Equation & Ref. & $U\left(10^{-21} \mathrm{~N} \mathrm{~m}\right)$ \\
\hline Yan et al. [21] & $U=\frac{6 \pi r_{1}^{r_{1}\left(\frac{r_{2}-r_{1}}{r_{1}}\right)^{2} B}}{(1+3 B / 4 \mu)}$ & $\begin{array}{c}\text { Eshelby } \\
{[22]}\end{array}$ & 5.7 \\
$\begin{array}{l}\text { Hwang and Chen } \\
\text { [9] }\end{array}$ & $\begin{array}{l}U=\frac{2}{9} \mu \Omega\left[\frac{(1+v)}{(1-v)}\right] \Gamma^{2} \\
\text { Eshelby } \\
{[23]}\end{array}$ & 6.9 \\
& & Avg. & 6.3
\end{tabular}

$\mu=$ shear modulus $(80 \mathrm{GPa}), \quad B=$ bulk modulus $(174 \mathrm{GPa})$, $\gamma=$ Poisson's ratio $(0.3), r_{1}=$ ionic radius of the host $(0.084 \mathrm{~nm})$, $r_{2}=$ ionic radius of the solute $(0.102 \mathrm{~nm}), \Gamma=$ Vegards slope for volume misfit $(0.077), \Omega=$ molecular volume $\left(3.5 \times 10^{-29} \mathrm{~m}^{3}\right)$ 
Regarding the electrostatic component of the GB energy, the number of excess (compared to the lattice) segregated aliovalent solute ions per unit GB area is

$N_{\mathrm{b}}^{\mathrm{s}}=\delta_{\mathrm{b}} \overline{\Delta C}_{\mathrm{b}} / \Omega \quad(\mathrm{m})^{-2}$

where $\overline{\Delta C}_{\mathrm{b}}$ is the integrated average excess segregated solute concentration. The corresponding electrostatic energy per unit GB area is then

$\gamma_{\mathrm{b}}^{\mathrm{e}}=N_{\mathrm{b}}^{\mathrm{s}} Z^{*} e \phi=\delta_{\mathrm{b}} \overline{\Delta C}_{\mathrm{b}} \Delta Z^{*} e \phi / \Omega \quad\left(\mathrm{eV} / \mathrm{m}^{2}\right)$

where $\Delta Z^{*}$ is the effective valence of the solute cation. Assuming that $\Delta \gamma_{\mathrm{b}}^{E}$ (in Eq. 2) results from the reduction in $\gamma_{\mathrm{b}}^{\mathrm{e}}$ by the applied field, Eq. 8 gives for the applied field $E$

$\Delta \gamma_{\mathrm{b}}^{E}=\gamma_{\mathrm{b}}^{\mathrm{e}}=\delta_{\mathrm{b}} \overline{\Delta C}_{\mathrm{b}} Z^{*} e \phi / \Omega \quad\left(\mathrm{eV} / \mathrm{m}^{2}\right)$

Taking $E=E_{\mathrm{c}}$ and rearranging Eq. 9 gives for the space charge potential energy due to the segregated aliovalent ions

$Z^{*} e \phi=\Delta \gamma_{\mathrm{b}}^{E_{\mathrm{c}}} \Omega / \delta_{\mathrm{b}} \overline{\Delta C_{\mathrm{b}}}$

where $E_{\mathrm{c}}$ is the critical field shown in Fig. 1 .

\section{Experimental}

Procedure

The starting material in the present isothermal annealing tests was 3 Y-TZP powder (GS $d_{0}=26 \mathrm{~nm}$ ) purchased from Tosoh having the following chemical composition in wt $\%$ :

\begin{tabular}{llllll}
\hline $\mathrm{Y}_{2} \mathrm{O}_{3}$ & $\mathrm{Al}_{2} \mathrm{O}_{3}$ & $\mathrm{SiO}_{2}$ & $\mathrm{Fe}_{2} \mathrm{O}_{3}$ & $\mathrm{Na}_{2} \mathrm{O}$ & $\mathrm{ZrO}_{2}$ \\
\hline 5.21 & $<0.005$ & 0.005 & 0.002 & 0.022 & Remainder \\
\hline
\end{tabular}

The powder compaction (98 MPa at room temperature), specimen geometry, and electrical connections for the present tests without and with electric field were the same as those employed previously [4]. The thermal history in the present tests is shown in Fig. 3. Following binder burn-out at $800{ }^{\circ} \mathrm{C}$ the specimens were furnace-cooled to room temperature and then sintered at a constant heating rate of $11^{\circ} \mathrm{C} /$ min without and with a constant applied dc voltage $V=25 \pm 0.1 \mathrm{~V}$ to $1300 \pm 1{ }^{\circ} \mathrm{C}$ and $1400 \pm 1{ }^{\circ} \mathrm{C}$ and then directly annealed at these temperatures for various times up to $24 \mathrm{~h}$, following which they were again furnace cooled. The linear shrinkage (optical measurement), which gave the relative density $\rho_{\mathrm{r}}$ and the corresponding GS $\bar{d}$ (SEM) were measured during the annealing. As a result of the shrinkage during sintering the applied electric field $E$ increased from the initial $14 \mathrm{~V} / \mathrm{cm}$ following binder removal to $\sim 18 \mathrm{~V} / \mathrm{cm}$ at the beginning of the isothermal anneal.

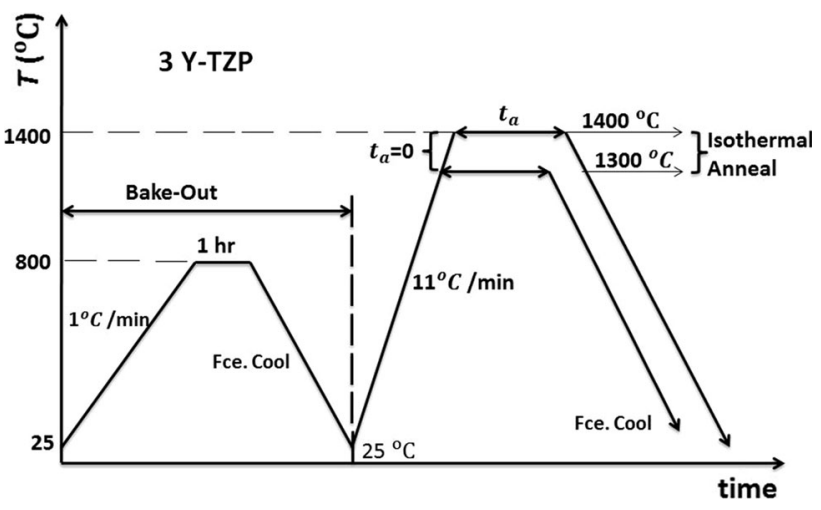

Fig. 3 Schematic of the thermal history of the specimens in the present isothermal annealing tests without and with dc electric field

For each annealing time, the mean linear intercept GS $\bar{d}$ was determined from SEM micrographs of mechanically polished and thermally etched $\left(1 \mathrm{~h}\right.$ at $\left.1200{ }^{\circ} \mathrm{C}\right)$ cross sections of the test specimens. The SEM micrographs were taken at three locations on each specimen: (a) $\sim 5 \mathrm{~mm}$ below the positive upper electrode, (b) midway between the two electrodes, and (c) $\sim 5 \mathrm{~mm}$ above the lower negative electrode. Approximately $200 \mathrm{GS}$ measurements were made at each location, giving a total of $\sim 600$ measurements for each annealing time. To establish the scatter in the measurements, the GS counts were made independently by three investigators for each of the three locations representing each annealing condition, giving a total of $\sim 1800$ counts. The GS reported is the mean value of the 1800 counts made independently by the three investigators; the reported scatter is the maximum and minimum values of the mean GS for each of the three locations.

An estimate of the grain shape anisotropy $\Gamma$ was taken to be the ratio of the mean GS determined from counts along lines parallel to the bottom edge of the SEM micrographs $\left(\bar{d}_{\mathrm{II}}\right)$ to those along perpendicular lines $\left(\bar{d}_{P}\right)$, i.e., $\Gamma=$ $\bar{d}_{\mathrm{II}} / \bar{d}_{P}$. Again, for each location and annealing condition $\Gamma$ was independently measured by the three investigators.

\section{Results}

An example of the grain structure in a specimen annealed with field compared to without is presented in Fig. 4. To be noted are: (a) the grains in both micrographs are relatively equiaxed and (b) the grains with field are significantly smaller than those without. Plots of $\rho_{\mathrm{r}}$ and $\bar{d}$ vs. annealing time $t_{\mathrm{a}}$ without and with applied electric field $(E=18 \mathrm{~V} /$ $\mathrm{cm})$ at 1300 and $1400{ }^{\circ} \mathrm{C}$ are presented in Fig. 5. The data points are the combined mean values for the three locations by the three investigators; the error bars are the maximum and minimum values of the means. To be noted in Fig. 5 is 
Fig. 4 SEM micrographs at the location midway between the electrodes of the specimens annealed for $12 \mathrm{~h}$ at $1300{ }^{\circ} \mathrm{C}$ : a $E=0$ and $\mathbf{b} E=18 \mathrm{~V} / \mathrm{cm}$
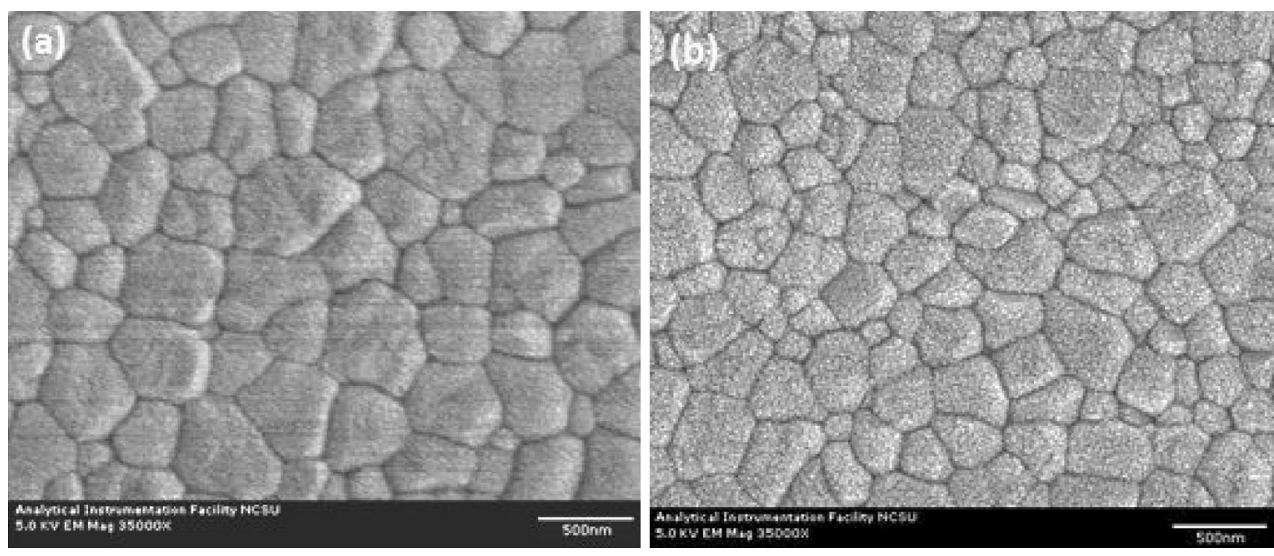

Fig. 5 Relative density $\rho_{\mathrm{r}}$ and mean linear intercept grain size $\bar{d}$ vs. annealing time $t_{\mathrm{a}}$ for the isothermal annealing of $3 \mathrm{Y}-\mathrm{TZP}$ without and with an applied dc electric field $E=18 \mathrm{~V} / \mathrm{cm}$ : a 1300 and b $1400{ }^{\circ} \mathrm{C}$

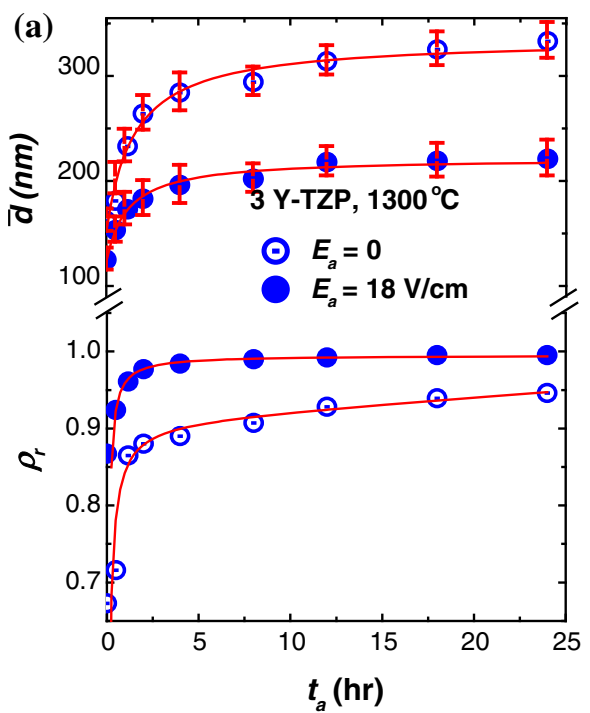

that $\bar{d}$ and $\rho_{\mathrm{r}}$ increase in a parabolic fashion with $t_{\mathrm{a}}$. At both temperatures, $\bar{d}$ decreases and $\rho_{\mathrm{r}}$ increases with the application of $E$. Further, the effect of $E$ on the difference $\Delta \bar{d}_{E}=\bar{d}-\bar{d}_{E}$ increases with annealing time. For all test conditions, the measured grain isotropy $\Gamma=1.05 \pm 0.05$, i.e., the grains were essentially isotropic. Further, there was no consistent trend in GS nor in $\Gamma$, regarding the three locations, i.e., from one electrode to the other.

As mentioned above, to avoid the need for the value of the driving force parameter $m$ in the solution of Eq. 3 one should know the magnitude of the GS ratio $\bar{d}_{E} / \bar{d}$ at a constant grain growth rate ratio $\bar{d}_{E} / \bar{d}$. For this, the values of $\dot{\bar{d}}_{E} / \dot{d}$ were determined from the slopes of the $\bar{d}$ vs. $t_{\mathrm{a}}$ curves in Fig. 5. The slopes (derived manually) are plotted vs. the reciprocal of the GS $(\bar{d})^{-1}$ in Fig. 6 . The hatched region defines where the magnitude of $\bar{d}_{E} / \bar{d}$ can be obtained at a constant $\bar{d}_{E} / \bar{d}$ and in turn for a corresponding constant driving force for grain growth. Also

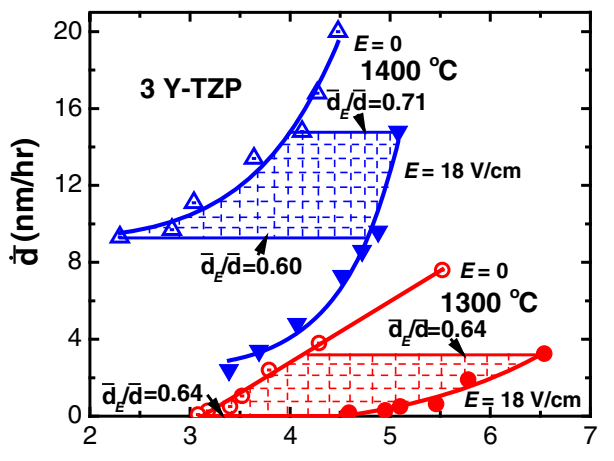

$(\bar{d})^{-1}(\mu \mathrm{m})^{-1}$

Fig. 6 Grain growth rate $(\bar{d})$ vs. the reciprocal of the grain size $(\bar{d})^{-1}$ for the present annealing tests without and with applied dc electric field $E=18 \mathrm{~V} / \mathrm{cm}$ at 1300 and $1400{ }^{\circ} \mathrm{C}$

shown in Fig. 6 are the values of the GS ratio $\bar{d}_{E} / \bar{d}$ at constant grain growth rate, which values range from 0.60 to 0.71 with an average of 0.65 . Of significance is that these values of $d_{E} / d$ for $E=18 \mathrm{~V} / \mathrm{cm}$ are in accord with those in 
Fig. 1 obtained at this field strength for other tests and processing conditions. The prior results (Fig. 1) and the present isothermal annealing test results thus give that the effect of an electric field on the GS ratio $\bar{d}_{E} / \bar{d}$ during sintering, plastic deformation and isothermal annealing is a unique function of the applied field strength, i.e., $\bar{d}_{E} / \bar{d}=$ $f(E)$ independent of $\bar{d}, T$, and $\rho_{\mathrm{r}}$. This in turn gives for a constant field strength that the difference in GS $\Delta \bar{d}_{E}=\bar{d}-\bar{d}_{E}=\bar{d}[1-f(E)]$, i.e., $\Delta \bar{d}_{E}$ is proportional to $\bar{d}$.

\section{Analysis and discussion}

The GB energy components $\gamma_{\mathrm{b}}^{\Sigma}$ and $\gamma_{\mathrm{b}}^{\mathrm{s}}$ represent elastic atomic displacements, which are usually essentially independent of an applied electric field $E$. The effect of $E$ on grain growth in 3Y-TZP is therefore entirely through its influence on the electrostatic component $\gamma_{\mathrm{b}}^{\mathrm{e}}$. The form of the $\bar{d}_{E} / \bar{d}$ vs. $E$ curve in Fig. 1 thus gives that the regime $E \leq E_{\mathrm{c}}$ corresponds to $\gamma_{\mathrm{b}}^{\mathrm{e}}$ and that for $E>E_{\mathrm{c}}$ to $\left(\gamma_{\mathrm{b}}^{\Sigma}-\gamma_{\mathrm{b}}^{\mathrm{s}}\right)$, where $\gamma_{\mathrm{b}}^{\mathrm{s}}$ is subtracted from $\gamma_{\mathrm{b}}^{\Sigma}$ because the ionic radius of $\mathrm{Y}^{3+}$ is larger than that of $\mathrm{Zr}^{4+}$ [9]. Therefore, since $d_{E} / d=$ 0.4 at $E=E_{\mathrm{c}}$, Eq. 3 gives $\gamma_{\mathrm{b}}^{\mathrm{e}}=0.6 \gamma_{\mathrm{b}}^{0}$, and in turn $\left(\gamma_{\mathrm{b}}^{\Sigma}-\gamma_{\mathrm{b}}^{\mathrm{s}}\right)=0.4 \gamma_{\mathrm{b}}^{0}$. Based on these magnitudes, we will compare the experimentally derived GB energy components with predictions.

Important in the evaluation of $\gamma_{\mathrm{b}}^{\Sigma}$ and $\gamma_{\mathrm{b}}^{\mathrm{s}}$ is a knowledge of their relative magnitudes. According to Eqs. 5 and 6 their ratio is given by

$\gamma_{\mathrm{b}}^{\Sigma} / \gamma_{\mathrm{b}}^{\mathrm{s}}=k_{\mathrm{B}} T / U C_{\mathrm{b}}^{*}$

Taking $T_{\text {avg }}=1350{ }^{\circ} \mathrm{C}, U=6.3 \times 10^{-21} \mathrm{~N}$ m (Table 1) and $C_{\mathrm{b}}^{*}=0.06$ [15], Eq. 11 gives $\gamma_{\mathrm{b}}^{\Sigma} / \gamma_{\mathrm{b}}^{\mathrm{s}}=59.3$, i.e., $\gamma_{\mathrm{b}}^{\Sigma} \gg \gamma_{\mathrm{b}}^{\mathrm{s}}$. The field-independent regime for $E>E_{\mathrm{c}}$ in Fig. 1 thus corresponds mainly to $\gamma_{\mathrm{b}}^{\Sigma}$, and therefore $\gamma_{\mathrm{b}}^{\Sigma} \approx 0.4 \gamma_{\mathrm{b}}^{0} \approx$ $0.254 \mathrm{~J} / \mathrm{m}^{2} \quad\left(\gamma_{\mathrm{b}}^{0}=1.215-0.358 \times 10^{-3} \mathrm{~T} \mathrm{~J} / \mathrm{m}^{2} \quad[24]\right)$. Setting $\gamma_{\mathrm{b}}^{\Sigma}=0.254 \mathrm{~J} / \mathrm{m}^{2}$, Eq. 5 gives $\lambda_{\mathrm{b}}^{\mathrm{c}}=1.21 \Omega^{1 / 3}$, which is not unreasonable considering: (a) the scatter in the data in Fig. 1, (b) the atomic structure in Fig. 2, and (c) general information on the width of grain boundaries in crystalline solids. Taking $\lambda_{\mathrm{b}}^{\mathrm{c}}=1.21 \Omega^{1 / 3}$, $U=6.3 \times 10^{-21} \mathrm{~N} \mathrm{~m} \quad$ (Table 1), and $C_{\mathrm{b}}^{*}=6 \times 10^{-2}$ [15], Eq. 6 gives $\gamma_{\mathrm{b}}^{\mathrm{s}}=4.3 \times 10^{-3} \mathrm{~J} / \mathrm{m}^{2}$. Thus, the difference $\left(\gamma_{\mathrm{b}}^{\Sigma}-\gamma_{\mathrm{b}}^{\mathrm{s}}\right) \approx 0.25 \mathrm{~J} / \mathrm{m}^{2}$, is in accord with that given by the field-independent regime $\left(E>E_{\mathrm{c}}\right)$ in Fig. 1. Moreover, the small value of $\gamma_{\mathrm{b}}^{\mathrm{s}}$ indicates that cation size misfit is not the major factor responsible for the segregation of yttria to the GBs in zirconia.

Regarding the electrostatic component $\gamma_{b}^{\mathrm{e}}$, according to Eq. $9 \Delta \gamma_{\mathrm{b}}^{\mathrm{e}}$ is proportional to $\Delta \gamma_{\mathrm{b}}^{E}$, the latter being given by Eq. 3. Employing Eq. 3 and taking $\gamma_{b}^{0}=0.63 \mathrm{~J} / \mathrm{m}^{2}$

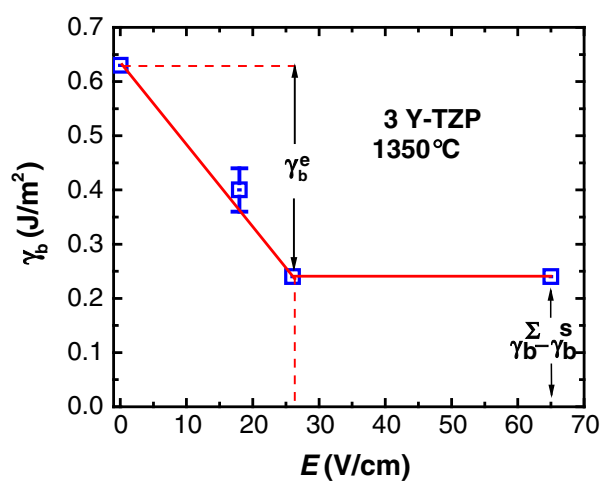

Fig. 7 Calculated effect of the applied dc field on the grain boundary energy in $3 \mathrm{Y}-\mathrm{TZP}$ at $T_{\text {avg }}=1350{ }^{\circ} \mathrm{C}$

[24] the effect of $E$ on $\Delta \gamma_{\mathrm{b}}^{E}$ at $1350{ }^{\circ} \mathrm{C}$ determined from the results in Figs. 1 and 6 is shown in Fig. 7. To be noted is that in keeping with Fig. 1 an approximately linear reduction in the GB energy by the applied field occurs up to the critical field $E_{\mathrm{c}} \cong 26 \mathrm{~V} / \mathrm{cm}$, with no further effect at higher fields to $65 \mathrm{~V} / \mathrm{cm}$. The rate of reduction in $\gamma_{\mathrm{b}}^{0}$ by the field $d \gamma_{\mathrm{b}}^{E} / d E$ is $1.46 \times 10^{-2}(\mathrm{~J} /$ $\left.\mathrm{m}^{2}\right) /(\mathrm{V} / \mathrm{cm})^{-1}$, and the total reduction of $\gamma_{\mathrm{b}}^{0}$ at $E_{\mathrm{c}}=26 \mathrm{~V} / \mathrm{cm}$ is $\sim 0.38 \mathrm{~J} / \mathrm{m}^{2}$. Thus, the major factor responsible for the segregation of yttria to the GBs in zirconia is electrostatic in nature, as proposed by Hwang and Chen [9]. Further, since the effect of electric field on grain growth in $3 \mathrm{Y}$-TZP is through its influence on the global GB energy (which per se is non-vectorial), it is expected that the field will not have a significant effect on the grain shape isotropy, as was in fact observed.

Of further interest regarding the electrostatic GB energy component $\gamma_{\mathrm{b}}^{\mathrm{e}}$ is a comparison of the magnitude of the space charge potential $\phi_{i}$ obtained from the present isothermal annealing tests with that determined by impedance spectroscopy and by atomic simulation. Taking for the parameters in Eqs. 9 and 10, $\Delta \gamma_{\mathrm{b}}^{E_{\mathrm{c}}}=0.60 \gamma_{\mathrm{b}}^{0}$ (Eq. 3), $\gamma_{\mathrm{b}}^{0}=0.63 \mathrm{~J} /$ $\mathrm{m}^{2}\left(T_{\text {avg }}=1350{ }^{\circ} \mathrm{C}[24]\right), \Omega=35 \times 10^{-30} \mathrm{~m}^{3}, \delta_{\mathrm{b}}=8 \mathrm{~nm}$ [15], and $\overline{\Delta C}_{\mathrm{b}}=1.6 \times 10^{-2}$ [15], one obtains $Z^{*} e \phi=$ $0.66 \mathrm{eV}$. Taking $\mathrm{Z}^{*}=3$ for the $\mathrm{Y}^{3+}$ ions gives $e \phi=0.22 \mathrm{eV}$. This value is in accord with those obtained by impedance spectroscopy [25-29] and atomic simulation [30]; see Table 2. Moreover, this value of $e \phi$ for 3Y-TZP is within the range reported for crystalline oxides in general [31-35]. These agreements provide support for the model proposed here for $\gamma_{b}^{\text {e }}$ (Eqs. 9 and 10) whereby the applied electric field exerts a bias on the space charge potential due to the segregation of $\mathrm{Y}^{3+}$ ions to the GBs; see Fig. 8.

It is well-known that $3 \mathrm{Y}-\mathrm{TZP}$ is an ionic conductor (due to the high mobility of the oxygen ions) and that the socalled "specific" GB resistivity $\rho_{\mathrm{b}}^{*}$ is one-to-three orders of the magnitude larger than that of the grain interior $\rho_{\mathrm{g}}[35-$ 
Table 2 Comparison of the space charge potential $\phi_{i}$ obtained in the present isothermal grain growth tests with those by the absolute reaction rate (ARR) theory, by impedance spectroscopy (IS) and by atomic simulation (AS)

\begin{tabular}{|c|c|c|c|c|c|c|}
\hline Authors & Material & Method & $d(\mu \mathrm{m})$ & $T\left({ }^{\circ} \mathrm{C}\right)$ & $\phi_{i}(V)$ & $\delta_{\mathrm{b}}(\mathrm{nm})$ \\
\hline Present & $3 \mathrm{YSZ}$ & Grain growth: $\dot{d}=$ const. & $0.1-0.5$ & $800-1500$ & 0.22 & 8.0 \\
\hline Conrad [5] & $3 \mathrm{YSZ}$ & Grain growth: ARR & $0.1-0.5$ & $800-1500$ & $0.12-0.18$ & - \\
\hline Verkerk et al. [25] & $8 \mathrm{YSZ}$ & IS & 0.28 & $600-1000$ & $0.20-0.23$ & 9.0 \\
\hline Guo and Maier [26] & $8 \mathrm{YSZ}$ & IS & 16 & $200-800$ & 0.25 & $\sim 5.0$ \\
\hline Guo [27] & $8 \mathrm{YSZ}$ & IS & 1.4 & 400 & 0.26 & 3.5 \\
\hline Guo et al. [28] & $8 \mathrm{YSZ}$ & IS & 16 & $250-500$ & $0.20-0.27$ & $\sim 4.0$ \\
\hline Guo and Zhang [29] & $3 \mathrm{YSZ}$ & IS & $0.12-1.3$ & $250-600$ & $0.18-0.24$ & 5.6 \\
\hline Guo and Zhang [29] & $8 \mathrm{YSZ}$ & IS & $0.25-13.5$ & 450 & $0.21-0.27$ & 4.8 \\
\hline Bingham et al. [30] & $6 \mathrm{YSZ}$ & AS & $<0.5$ & 1700 & $0.15-0.22$ & 1.7 \\
\hline
\end{tabular}

$d=$ grain size, $\phi_{i}=$ space charge potential, $\delta_{\mathrm{b}}=$ total grain boundary width

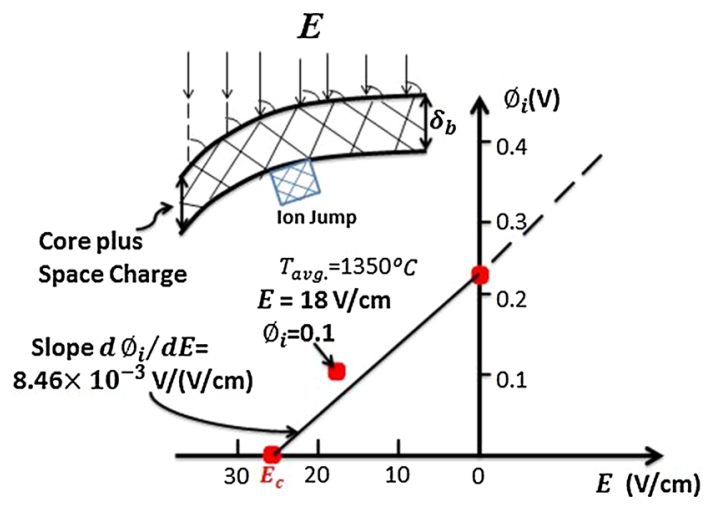

Fig. 8 The bias exerted at $1350{ }^{\circ} \mathrm{C}$ by the applied dc electric field $E$ on the space charge potential $\phi_{i}$ in 3 YSZ due to the segregation of yttrium ions at the grain boundary

37].The relationship between the mechanism by which an electric field retards grain growth and that which governs $\rho_{\mathrm{b}}^{*}$ has yet to be determined. The present results, along with those obtained previously (Fig. 1), give that the mechanism by which a field retards grain growth is by reducing the relatively athermal GB energy through the effect of the field on the space charge potential. The higher $\rho_{\mathrm{b}}^{*}$ compared to $\rho_{\mathrm{g}}$ could be due to a reduction in the oxygen ion mobility through the GB as a result of reduction in the oxygen ion vacancy concentration. The mechanism by which a reduction in the oxygen vacancies might occur is presently under investigation by the authors.

The contribution of the space charge $\phi_{i}$ to $\gamma_{\mathrm{b}}^{0}$ obtained from cross plots of the results in Figs. 7 and 8 is shown in Fig. 9. The variation of $\gamma_{\mathrm{b}}$ with $\phi_{i}$ in Fig. 9 thus gives that any physical property which is a function of $\gamma_{b}$ (e.g., grain growth rate or plastic strain rate) will depend on the valence of the solute as well as its ionic size misfit. Thus, properties relating to $\gamma_{\mathrm{b}}$ can be varied by the addition of selected aliovalent solutes and the application of an electric field.

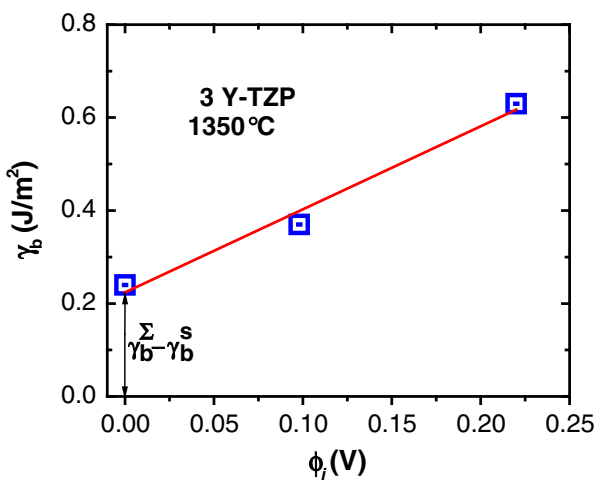

Fig. 9 Grain boundary energy $\gamma_{\mathrm{b}}$ vs. the magnitudes of the space charge potential $\phi_{i}$ in $3 \mathrm{YSZ}$ at $1350{ }^{\circ} \mathrm{C}$

\section{Summary and conclusions}

The effect of an applied dc electric field $E$ up to $\sim 60 \mathrm{~V} / \mathrm{cm}$ on grain growth in 3Y-TZP shows two regimes: (a) $E \leq E_{\mathrm{c}}(26 \mathrm{~V} / \mathrm{cm})$, where the GS ratio with field compared to without $\left(d_{E} / d\right)$ decreases with $E$ and (b) $E>E_{\mathrm{c}}$ where $d_{E} / d$ is essentially independent of $E$, the transition occurring at $d_{E} / d \approx 0.4$. It is proposed that the former regime corresponds to the electrostatic (space charge) component $\gamma_{b}^{\mathrm{e}}$ of the GB energy and the latter to the difference between the crystallographic $\left(\gamma_{\mathrm{b}}^{\Sigma}\right)$ and the size misfit $\left(\gamma_{\mathrm{b}}^{\mathrm{s}}\right)$ components. The electrostatic component accounts for $60 \%$ of the total GB energy and the crystallographic and cation size misfits for the remaining $40 \%$.

A physical model is developed for determining the magnitudes of the three GB energy components in 3Y-TZP from the effect of an electric field on grain growth. Reasonable agreement occurred between the experimentally derived values of the GB energy components and predictions. Moreover, the derived value of the space charge potential $e \phi_{i}=0.22 \mathrm{eV}$ was in accord with reported values from impedance spectroscopy and atomic simulation. 
The present results support the idea that the segregation of yttria to the grain boundaries in 3Y-TZP is mainly due to an electrostatic factor and that the resulting retardation of grain growth by an electric field is mainly due to a reduction in the GB energy, i.e., in the capillary driving force.

Acknowledgements This research was funded by NSF Grant No. DMR-1002751, Dr. Lynnette Madsen, Manager Ceramics Program, Materials Science Division. The authors wish to thank Dr. Di Yang and Ms. A. H. Du for their contributions to the grain size measurements.

\section{References}

1. Kingery WD, Bowen HK, Uhlmann DR (1996) Introduction to ceramics, 2nd edn. Wiley, New York

2. Yang Di, Conrad H (1997) Influence of an electric field on the superplastic deformation of 3Y-TZP. Scr Mater 36:1431-1435

3. Ghosh S, Chokshi AH, Lee P, Raj R (2009) A huge effect of weak electric fields on grain growth in zirconia. J Am Ceram Soc 92:1856-1859

4. Conrad H, Yang Di (2011) Dependence of the sintering rate and related grain size of yttria-stabilized zirconia (3Y-TZP) on the strength of an applied DC electric field. Mater Sci Eng A 528:8523-8529

5. Conrad H (2012) Retardation of grain growth in nanocrystalline zirconia by an electric field. Emerg Mater Res Inst Civil Eng $1: 11-16$

6. Starnes S, Conrad H (2008) Grain size distribution in ultrafinegrained yttria-stabilized zirconia deformed without and with an electric field. Scr Mater 59:1115-1118

7. Obare J, Griffin WD, Conrad H (2012) Effect of heating rate and DC electric field on the grain size distribution in fully-sintered zirconia polycrystals stabilized with $3 \mathrm{~mol} \%$ yttria (3Y-TZP). J Mater Sci 47:5141-5147. doi:10.1007/s10853-012-6391-5

8. Winnubst AA, Kroot PJM, Burggraaf AJ (1983) AES/STEM grain boundary analysis of stabilized zirconia ceramics. J Phys Chem Solids 44:955-960

9. Hwang S-L, Chen I-W (1990) Grain size control of tetragonal zirconia polycrystals using the space charge concept. J Am Ceram Soc 73:3269-3277

10. Theunissen GSAM, Winnubst AJA, Burggraaf AJ (1992) Surface and grain boundary analysis of doped zirconia ceramics studied by AES and XPS. J Mater Sci 27:5057-5066. doi:10.1007/ BF01105274

11. Stemmer S, Vdeugels J, Vand der Beist O (1998) Grain boundary segregation in high-purity, yttria-stabilized tetragonal zirconia polycrystals (Y-TZP). J Eur Ceram Soc 18:1565-1570

12. Hines JA, Ikuhara Y, Chokshi AH, Sakuma T (1998) The influence of trace impurities on the mechanical characteristics of a superplastic $2 \mathrm{~mol} \%$ yttria stabilized zirconia. Acta Mater 46:5557-5568

13. Dickey EC, Fan Z, Pennyeook SJ (2001) Structure and chemistry of yttria-stabilized cubic-zirconia symmetric tilt grain boundaries. J Am Ceram Soc 84:1361-1368

14. Flewitt PEJ, Wild RK (2001) Grain boundaries: their microstructure and chemistry. Wiley, Chichester

15. Matsui K, Yashida H, Ikuhara Y (2008) Grain boundary structure and microstructure development mechanism in 2-8 mol\% yttriastabilized zirconia polycrystals. Acta Mater 56:1315-1325
16. Chaim R (2008) Activation energy and grain growth in nanocrystalline Y-TZP ceramics. Mater Sci Eng A 486:439-446

17. Cahn JW (1962) The impurity-drag effect in grain boundary motion. Acta Metall 10:789-798

18. Yan MF, Cannon R, Bowen H (1977) Grain boundary migration in ceramics. In: Fulrath RM, Pask JA (eds) Ceramic Microstructures, II processing, Westview Press, Boulder, pp 276-307

19. Wang J, Yang Di, Conrad H (2013) Transient-regime grain growth in nanocrystalline yttria-stabilized zirconia annealed without and with a DC electric field. Scr Mater 69:351-353

20. Gottstein G, Shvindlerman LS (1999) Grain boundary migration in metals. CRC Press, New York, p 112

21. Yan MF, Cannon RM, Bowen HK (1983) Space charge, electric field, and dipole contributions to equilibrium solute segregation at interfaces. J Appl Phys 54:764-776

22. Eshelby JD (1956) The continuum theory of lattice defects. In: Seitz F, Turnbull D (eds) Solid state physics. Academic Press, New York

23. Eshelby JD (1957) Determination of the elastic field of an ellipsoidal inclusion and the related problems. Proc R Soc Lond A 241:376-396

24. Tsoga A, Nikolopoulos P (1996) Surface and grain boundary energies in yttria-stabilized zirconia (YSZ-8 mol \%). J Mater Sci 31:5409-5413. doi:10.1007/BF01159310

25. Verkerk MJ, Middelhuis BJ, Burgraff AJ (1982) Effect of grain boundaries on the conductivity of high-purity $\mathrm{ZrO}_{2}-\mathrm{Y}_{2} \mathrm{O}_{3}$ ceramics. Solid State Ionics 6:159-170

26. Guo X, Maier J (2001) Grain boundary blocking effect in zirconia: a Schottky barrier analysis. J Electrochem Soc 148:E121E126

27. Guo X (2001) Defect structure modification in zirconia by alumina. Phys Stat Sol (a) 183:261-271

28. Guo X, Sigle W, Fleig J, Maier J (2002) Role of space charge in the grain boundary blocking effect in doped zirconia. Solid State Ionics 154-155:555-561

29. Guo X, Zhang Z (2003) Grain size dependent grain boundary defect structure: case of doped zirconia. Acta Mater 51:2539-2547

30. Bingham D, Tasker PW, Cormack AN (1989) Simulated grainboundary structures and ionic conductivity in tetragonal zirconia. Phil Mag A 60:1-14

31. Kingery WD (1974) Plausible concepts necessary and sufficient for interpretation of ceramic grain-boundary phenomena: I. Grain-boundary characteristics, structure and electrostatic potential. J Am Ceram Soc 57:1-8

32. Ikeda JA, Chiang Y-M (1993) Space charge segregations at grain boundaries in titanium oxide: I. Relationship between lattice defect chemistry and space charge potential. J Am Ceram Soc 76:2437-2446

33. Huey BD, Lisjak D, Bonnell DA (1999) Nanometer-scale variations in interface potential by scanning probe microscopy. J Am Ceram Soc 82:1941-1944

34. Han J-H, Kim D-Y (2001) Interactions and chemistry of defects at the grain boundaries of ceramics. J Am Ceram Soc 84:539-550

35. Guo X, Wasser R (2006) Electrical properties of the grain boundaries of oxygen ion conductors: acceptor-doped zirconia and ceria. Progr Mater Sci 51:151-210

36. Wang J, Du A, Yang D, Raj R, Conrad H (2013) Grain boundary resistivity of yttria-stabilized zirconia at $1400^{\circ} \mathrm{C}$. J Ceram Article ID 370603:1-4

37. Wang J, Conrad H (2013) Grain boundary resistivity in yttriastabilized zirconia. In: Bansai NP et al (eds) Processing and properties of advanced ceramics and composites, vol 240. Wiley, New York, pp 175-188 